\title{
Performance Analysis of Daubechies Wavelet in Image Deblurring and Denoising
}

\author{
Raj Ranjan Singh ${ }^{1}$, Satyabrata Das ${ }^{2}$ \\ ${ }^{1}$ (M.Tech. ECE $2^{\text {nd }}$ year,National Institute of Science and Technology, Berhampur,Odisha, India) \\ ${ }^{2}$ (Associate professor, National Institute of Science and Technology, Berhampur, Odisha, India)
}

\begin{abstract}
Images get blurred when they are acquired and contaminated by noise while being transmitted,hence it is necessary to restore the image and remove the noise present in the image. The deconvoluion is performed for removing blur from the image in many application such as astronomy, remote sensing and medical imaging etc.In this paper we have used wavelet transform to deblur the image and Daubechies wavelet is used for this purpose.We have compared the performance of wavelet based deconvolution with the Weiner deconvolution method. The simulation has been performed by contaminating the image using different types of blur and signal to noise ratio in both cases have been calculated .
\end{abstract}

Keywords: Daubechies wavelet, signal to noise ratio, wavelet transform, wavelet based deconvolution, Weiner deconvolution

\section{INTRODUCTION}

The image deconvolution or deblurring is the task of recovery of the original image from the observed corrupted image. The image is generated from physical process, its values are proportional to energy radiated by a physical source. The resultant image $\mathrm{i}(\mathrm{x}, \mathrm{y})$ is non-zero and finite[1].

$$
\mathrm{i}(\mathrm{x}, \mathrm{y}) \in \mathrm{Z}
$$

Where $\mathrm{Z}$ is finite set of integers and $\mathrm{x}, \mathrm{y}$ denotes spatial coordinates. The image is interpreted as two dimensional light intensity function $\mathrm{i}(\mathrm{x}, \mathrm{y})$ and the value $\mathrm{i}$, at any point $(\mathrm{x}, \mathrm{y})$ is proportional to the brightness (or gray level ) of image at that point[1].A digital image may be considered as matrix whose row and column indices represents point in the image and the corresponding matrix element known as picture element, pixels values identifies gray level at that point. The image formation depends on the characteristics of the object being captured, environmental conditions and the image capturing system. The process of reconstructing the original image from the degraded version is known as image restoration. The image degradation model is represented as

$$
d=h_{f} i+n
$$

Where $\mathrm{d}$ is degraded image, $\mathrm{h}_{\mathrm{f}}$ is the degradation function known as point spread function(PSF) or blur and $\mathrm{n}$ is the additive noise. For given $\mathrm{d}$, some knowledge about the degradation function $\mathrm{h}_{\mathrm{f}}$ and some knowledge about $n$,the objective of the restoration is to obtain an estimate of original image. The process includes deblurring of images by the limitation of a sensor and its environment, noise filtering and correction of geometric distortion or non-linearity due to sensor. The image restoration has many application in astronomical imaging, medical imaging, remote sensing and it has found application in x-ray imaging.

The recovery process can be sub divided into two categories:

(i) Classical Restoration

(ii) Blind image restoration or deconvolution

Classical image restoration includes the techniques which utilizes some prior information about the PSF during reconstruction.In blind image deconvolution an observed or degraded image $\mathrm{d}(\mathrm{x}, \mathrm{y})$ is assumed to be two dimensional convolution of true image $\mathrm{i}(\mathrm{x}, \mathrm{y})$ with linear shift invariant blur known as PSF $\mathrm{h}(\mathrm{x}, \mathrm{y})$ and additive noise is assumed zero[2].

$$
d(x, y)=i(x, y) * h(x, y)
$$

The problem of reconstructing the true image $\mathrm{i}(\mathrm{x}, \mathrm{y})$ requires the deconvolution of the PSF $\mathrm{h}(\mathrm{x}, \mathrm{y})$ from the degraded image $\mathrm{d}(\mathrm{x}, \mathrm{y})$.Many research has been done exploring various methods for image deconvolution,but it is one of the challenging problem for the researcher. Authors of [3] have demonstrated a novel way of wavelet decomposition to sub sample and image to abstract information for automatic decision making. Authors 
of [4] has proposed an efficient, hybrid Fourier-wavelet regularized deconvolution (ForWaRD) algorithm that performs noise regularization via scalar shrinkage in both the Fourier and wavelet domains.

In this paper we have shown the performance of daubechies wavelet based deconvolution for removing the blur from the degraded image.

\section{WAVELET TRANSFORM}

The wavelet transform decomposes a signal into set of basis function. In the wavelet transform the basis function is [5].

$$
\psi_{a, b}(x)=\frac{1}{\sqrt{a}} \psi\left(\frac{x-b}{a}\right)
$$

Where 'a' represents scale variable and the variable 'b' represents time shift or translation. Since the wavelet transform is generated using dilates and translates of the single function $\psi(t)$, the wavelet for the transform is referred to as mother wavelet.

The discrete wavelet transform represents a 1-d signal $\mathrm{x}$ in terms of shifted versions of a low-pass scaling function $\phi$ and shifted and dilated versions of a prototype bandpass wavelet function $\psi$.For special choices of $\phi$ and $\psi$, the functions [6]

$$
\begin{aligned}
\psi_{j, k}(t) & :=2^{j / 2} \psi\left(2^{j} t-k\right) \\
\phi_{j, k}(t) & :=2^{j} \phi\left(2^{j} t-k\right), j, k \in \mathbf{Z}
\end{aligned}
$$

Form an orthonormal basis, and we have the representation [6]

$$
\begin{gathered}
x(t)=\sum_{k} u_{j_{0}, k} \phi_{j_{0}, k}(t)+\sum_{j=j_{0}}^{j} \sum_{k} w_{j, k} \psi_{j, k}(t) \\
w_{j, k}:=\int x(t) \psi_{j, k}^{*}(t) d t \\
u_{j, k}:=\int x(t) \phi_{j, k}^{*}(t) d t
\end{gathered}
$$

The parameter $\mathbf{J}$ controls the resolution of wavelet representation. For a discrete-time signal, the $\mathrm{n}$ wavelet coefficients $\left\{\mathrm{u}_{\mathrm{j} 0}, \mathrm{w}_{\mathrm{j}, \mathrm{k}}\right\}$ of $\mathrm{x}\left(\mathrm{t}_{\mathrm{n}}\right)$ can be easily computed using a filter bank consisting of low pass filters, high pass filters, and decimators[6]. Due to the special filter band structure the forward and inverse wavelet transform can be computed in $O(\mathrm{~N})$ operations, where $\mathrm{N}$ is the length of the signal.

\subsection{Multiresolution Analysis}

In discrete data domain, the resolution of the data is the level of detail. Since the wavelet transform can be analyzed at different side, the different resolution of wavelet domain can be obtained and a shift invariant interpretation can be performed. In brief, forward wavelet transform (FWT) for input signal $\mathrm{x}$ in $\mathrm{j}^{\text {th }}$ level by multiresolution analysis(MRA) and its inverse wavelet transform (IWT) can simply expressed using convolution as follows;

$$
\begin{gathered}
w_{j+1}=l * v_{j} \\
v_{j+1}=k^{*} v_{j} \\
v_{j}=k^{*} v_{j+1}+l * w_{j+1}
\end{gathered}
$$

where $w_{j}$ and $v_{j}$ are vector for wavelet and scaling coefficients in $j^{\text {th }}$ level,$k$ and 1 are scaling and wavelet filters. $\mathrm{w}_{\mathrm{j}}$ and $\mathrm{v}_{\mathrm{j}}$ can be down-sampled for FWT and up-sampled for IWT. As proven by Daubechies [7],the input data decomposed by down-sampling can be reconstructed without any loss.

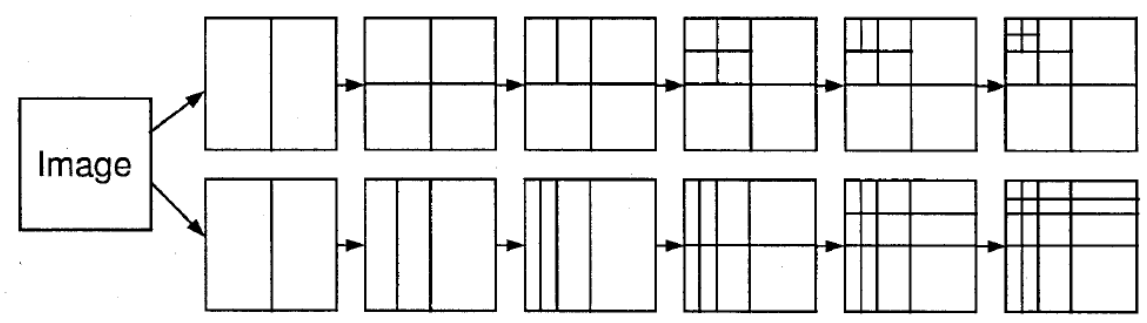

Fig. 2 Two different 2D separable wavelet transform approaches (upper: square-shaped approach, lower: rectangular approach) 


\subsection{Two dimensional Wavelet Transform}

There are several ways to perform two-dimensional (2D) wavelet transform such as non-separable transform[8] rectangular separable transform[9] and squared-shaped separable approach. Most popular is square separable approach by applying two 1D operations for all rows and then all columns for each decomposition levels.2D FWT for the $\mathrm{j}^{\text {th }}$ level can be obtained in similar way to $1 \mathrm{D}$ FWT .In $2 \mathrm{~d}$ wavelet domain shown in fig.3., each square shaped set of wavelet coefficients are called a sub band. In 2D image, each decomposition level can have four filtered sub bands, $\mathrm{LL}, \mathrm{LH}, \mathrm{HL}$ and $\mathrm{HH}$. The sub band labeled $\mathrm{LH}_{1}, \mathrm{HL}_{1}$, and $\mathrm{HH}_{1}$ are sets of wavelet coefficients in finest level. The following coarser level of sub bands, $\mathrm{LH}_{2}, \mathrm{HL}_{2}$ and $\mathrm{HH}_{2}$ are obtained from the scaling coefficients in the finer level $\mathrm{LL}_{1}$. The sub band and its adjacent coarser level of sub bands generated by the same filter have the parent-child relationship.

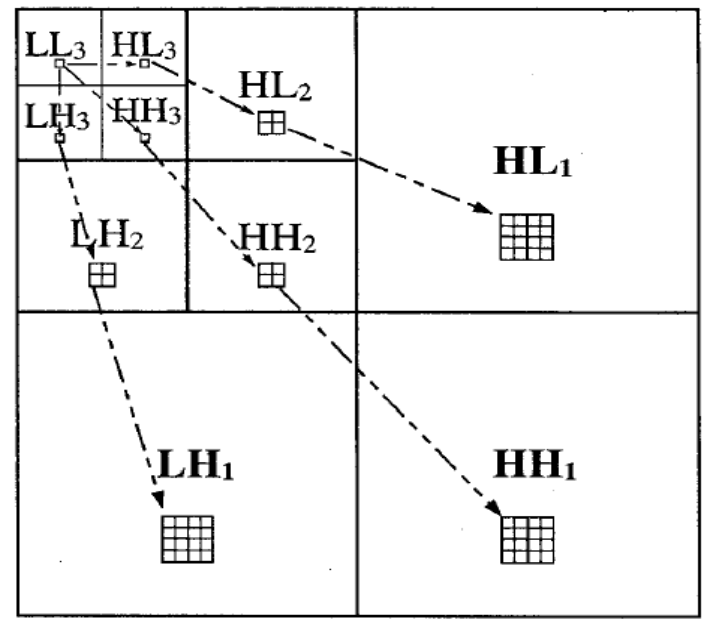

Fig.3 2D separable wavelet transform

\section{WEINER DECONVOLUTION}

The optimal weights that can be used to shrink each Fourier components during regularized inversion is a function of the SNR at each frequency. For wide-sense stationary signals, the optimal weights are given by [10],

$$
R\left(f_{n}\right):=\frac{\left|H\left(f_{n}\right)\right|^{2} P_{x}\left(f_{n}\right)}{\left|H\left(f_{n}\right)\right|^{2} P_{x}\left(f_{n}\right)+\sigma^{2}}
$$

Where $P_{x}\left(f_{n}\right)$ is the power spectral density of the stochastic signal $\mathrm{x}$ and $\sigma^{2}$ is the variance of the additive noise. The resulting LTI quasi-inverse operator is called the LTI Weiner deconvolution filter.

Inversion- The noisy and blurred observation $\mathrm{d}$ is treated with $\mathrm{H}^{-1}$ to obtain noisy, unbiased estimate $\mathrm{i}$ of the input signal .This step necessarily amplifies the noise components at frequencies where $\mathrm{H}\left(\mathrm{f}_{\mathrm{n}}\right)$ is small. Further processing is required to estimate the signal from the amplified noise.

Fourier domain signal estimation - Each frequency component of the noisy signal obtained after pure inversion is shrunk using weights $R\left(f_{n}\right)$.), it is clear that less shrinkage is employed at components where the SNR is high . The LTI Weiner deconvolution estimates the input signal is

$$
\hat{I}\left(f_{n}\right):=R\left(f_{n}\right) I\left(f_{n}\right)+R\left(f_{n}\right)\left(\frac{1}{H\left(f_{n}\right)}\right) \Gamma\left(f_{n}\right)
$$

For Gaussian WSS signals, the LTI Weiner deconvolution provides globally MSE-optimal estimate of the input since the Fourier domain ideally represents both, the noise after inversion, and the signal of interest. The Fourier coefficients of the signal and the noise by $\mathrm{H}^{-1}$ are independent making individual, scalar estimation in the Fourier domain optimal. However, when the input signal or noise is not Gaussian, Weiner filtering no longer remains optimal.

IV. DEBLURRING ALGORITHM

The deblurring algorithms[4] using $\mathrm{db}$ wavelet is:

(i) The input image (256x256) is selected from the file and then it is blurred with PSF of different size.

(ii) The two wavelet filters of different length were simulated to perform the estimation. 
(iii) The thresholding factor was set, and decomposition level is selected.

(iv) Then Wiener estimation is performed.

(v) Regularized inverse filtering is done on the degraded image, Fourier processing is performed.

(vi) Wavelet domain estimation of true image is performed.

\section{SIMULATION RESULTS}

The daubechies scaling function for filter of length 8 and 20 with minimum phase simulated is shown below:

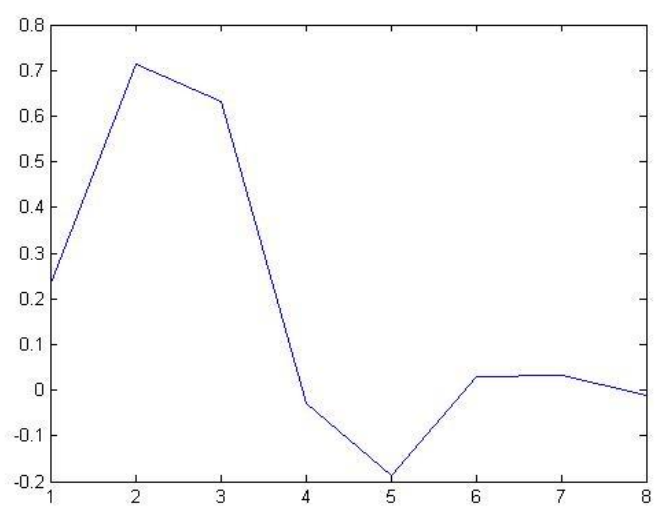

Fig.4 db scaling for filter of length 8

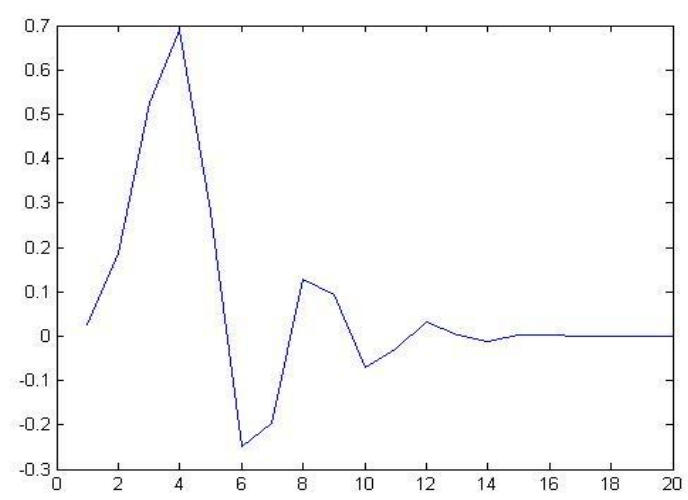

Fig. $5 \mathrm{db}$ scaling function for filter of length 20

The wavelet based deconvolution algorithm was implemented using MATLAB 7.10.Daubechies wavelet has been used in the algorithm for the deblurring the images. We have taken two images for consideration,the first image is 256 x 256 boat image and second image is $256 \times 256$ text image(a synthetically generated binary text image of the word "DAUBECHIES WAVELET". We have set the noise variance such that the BSNR is $40 \mathrm{~dB}$ and regularization parameter is set equal to 1.Firstly the boat image is blurred by 2-D 9x9 blur, then the same image is blurred with 2-D 9x9 blur)and this image is also blurred by circular blur .Next the NIST image is blurred by 2-D 9x9 blur then the same image is blurred with 2-D 9x9 blur and this image is also blurred by circular blur of radius 7.The summary of the simulation results is given below table:

Table.1: Simulation results

\begin{tabular}{|l|l|l|l|l|l|}
\hline \multirow{4}{*}{ Image } & \multirow{2}{*}{$\begin{array}{l}\text { Blur } \\
\text { BSNR=40dB }\end{array}$} & \multicolumn{2}{|l|}{ Weiner Estimate } & \multicolumn{2}{l|}{ Wavelet Estimate } \\
\cline { 3 - 6 } & & ISNR(dB) & SNR(dB) & ISNR(dB) & SNR(dB) \\
\hline \multirow{3}{*}{ BOAT } & $9 \times 9$ & 6.26 & 23.29 & 9.05 & 26.09 \\
\cline { 2 - 6 } & $21 \times 21$ & 7.78 & 21.44 & 8.69 & 22.34 \\
\cline { 2 - 6 } & circular & 9.19 & 23.4 & 13.38 & 27.59 \\
\hline \multirow{3}{*}{$\begin{array}{l}\text { TEXT } \\
\text { IMAGE }\end{array}$} & $9 \times 9$ & 7.71 & 12.12 & 12.3 & 16.7 \\
\cline { 2 - 6 } & $21 \times 21$ & 6.86 & 8.67 & 8.74 & 10.56 \\
\cline { 2 - 6 } & circular & 8.46 & 13.4 & 17.63 & 22.57 \\
\hline
\end{tabular}




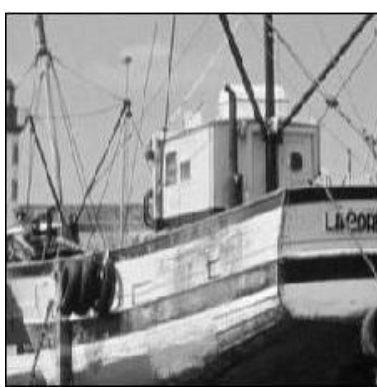

(a) Original image

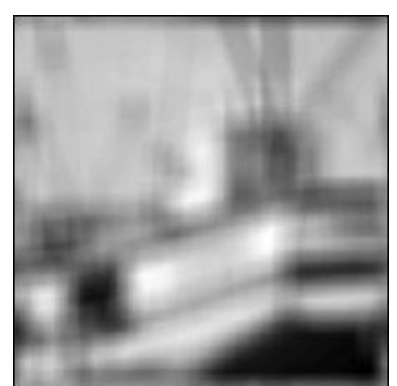

(b) Blurred image

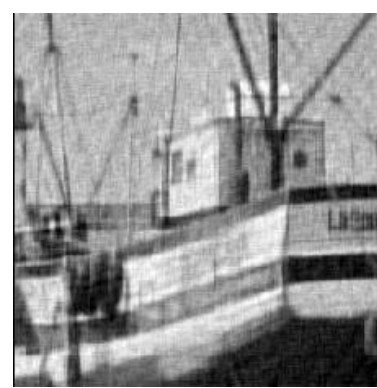

(c) Weiner estimate

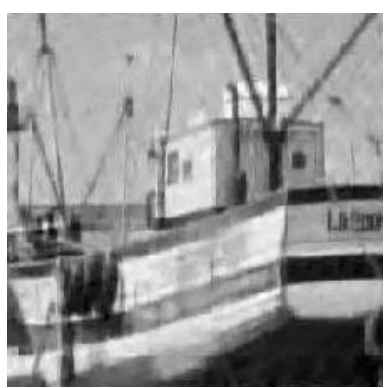

(d) Wavelet estimate

Fig.6 Results for the Boat image degraded by the $21 \times 21$ boxcar blur for a BSNR of $40 \mathrm{~dB}$.

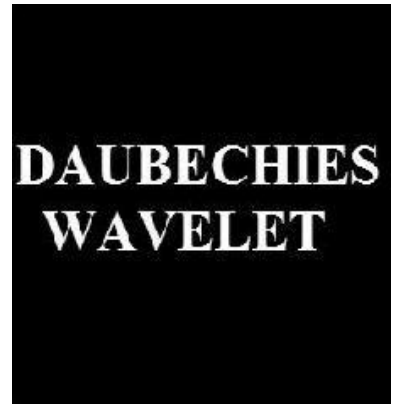

(a) Original image

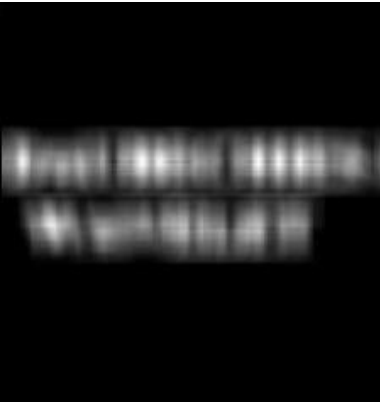

(b) Blurred image

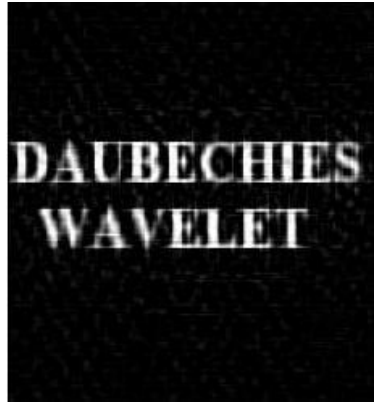

(c) Weiner estimate

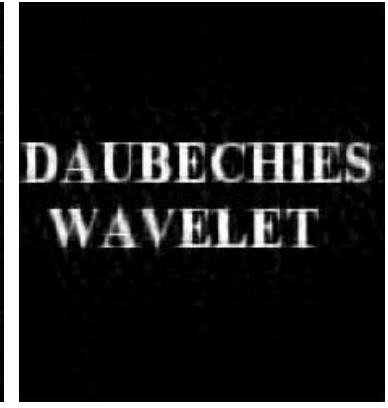

(d) Wavelet estimate

Fig.7 Results for the text image degraded by the 21x21 blur for a BSNR of $40 \mathrm{~dB}$.

VI.

CONCLUSION

From the simulation results, we conclude that the performance of the image debluring by using wavelet technique is better as compared to the Weiner deconvolution method as the signal to noise ratio and the improved signal to noise ratio obtained in wavelet case is higher than that in the Wiener case .The proper selection of the regularization parameter in the algorithm is necessary for obtaining better results.

\section{REFERENCES}

[1] Jain Anil K., Fundamentals of Digital Image Processing, Davis: Prentice-Hall of India, 2000.

[2] D. Kundur and D. Hatzinakos, Blind image deconvolution, IEEE Signal Processing Magazine, vol. 13, no. 3, pp. 43-64, Feb. 1996.

[3] P.Premaratne and I.Burnett, Role of wavelet transforms in image restoration, TENCON 2004. IEEE Regio10 Conference, vol. A, 243-246., 21-24 November 2004.

[4] R.Neelamani, H.Choi and R.Baraniuk, FoRWaRD: Fourier-Wavelet Regularized Deconvolution for IllConditioned Systems, IEEE Transactions on Signal Processing, vol. 52, no. 2, pp. 418-433, Feb.2004.

[5] Raghuveer M. Rao and Ajit S. Bopardikar, Wavelet Transforms-Introduction to theory and applications,Pearson Education 2002.

[6] S. Mallat, A Wavelet Tour of Signal Processing. New York: Academic, 1998

[7] I.Daubechies, Ten Lectures On Wavelets, SIAM, 1992.

[8] E.P Simoncelli and E.H. Edelson,Non-separable extensions of quadrature mirror filters to multiple Dimensions, Proceedings of the IEEE, 78(4):652-664, 1990.

[9] M.Jansen, Noise Reduction byWavelet Thresholding, Series: Lecture Notes in Statistics, Vol.161, Springer, New York, 1991.

[10] Ramesh Neelamani, Wavelet-Based Deconvolution for Ill-Conditioned Systems, Master of Science thesis, Rice University, Houston, Texas, USA, May1999. 